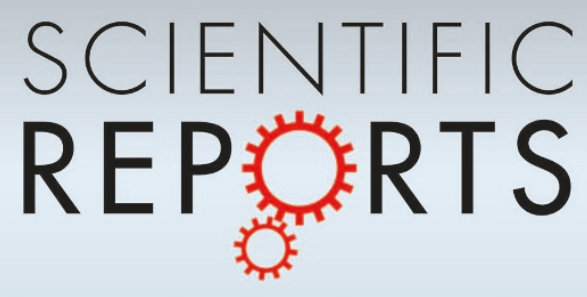

OPEN

SUBJECT AREAS:

RISK FACTORS

LIPOPROTEINS

CARDIOVASCULAR DISEASES

DIABETES

Received

22 April 2014

Accepted

21 May 2014

Published

17 June 2014

Correspondence and requests for materials should be addressed to

M.M. (marianne.i. mansson@ astrazeneca.com) or W.K. (Wolfgang. Knecht@biol.lu.se)

* Current address: Department of Biology, Molecular Cell Biology, Lund University, 22362 Lund, Sweden

\section{Lp(a) is not associated with diabetes but affects fibrinolysis and clot structure ex vivo}

Marianne Månsson' ', Inge Kalies', Göran Bergström², Caroline Schmidt², Anne Legnehed', Lillemor Mattsson Hultén ${ }^{2}$, Lena Amrot-Fors' ', David Gustafsson' \& Wolfgang Knecht * 'AstraZeneca R\&D Mölndal, 43183 Mölndal, Sweden, ${ }^{2}$ Wallenberg Laboratory for Cardiovascular Research, Sahlgrenska
Academy, University of Gothenburg, S-41345 Göteborg, Sweden.

Lipoprotein (a) $[\mathrm{Lp}(\mathrm{a})]$ is a low density lipoprotein (LDL) with one apolipoprotein (a) molecule bound to the apolipoprotein B-100 of LDL. $\operatorname{Lp}(a)$ is an independent risk factor for cardiovascular disease (CVD).

However, the relationship of $L p(a)$ to diabetes and metabolic syndrome, both known for increased CVD risk, is controversial. In a population based study on type two diabetes mellitus (T2DM) development in women, $\mathrm{Lp}(\mathrm{a})$ plasma levels showed the well known skewed distribution without any relation to diabetes or impaired glucose tolerance. A modified clot lysis assay on a subset of 274 subjects showed significantly increased clot lysis times in T2DM subjects, despite inhibition of PAI-1 and TAFI. Lp(a) plasma levels significantly increased the maximal peak height of the clot lysis curve, indicating a change in clot structure. In this study $\mathrm{Lp}(\mathrm{a})$ is not related to the development of T2DM but may affect clot structure ex vivo without a prolongation of the clot lysis time.

ipoprotein(a) [Lp(a)] is a circulating lipoprotein composed of liver-derived apolipoprotein(a) [apo(a)] covalently bound to apolipoprotein B-100 of low density lipoprotein (LDL). The LPA gene encoding the - apo(a) protein has evolved by duplication from the PLG gene, encoding plasminogen. Many of the potential pathological properties of $\operatorname{Lp}(\mathrm{a})$ have been attributed to the main structural domains of apo(a), the so called Kringles. The current understanding of the physiological and pathophysiological role of $L p(a)$ has recently been reviewed ${ }^{1}$. The concentration of $\mathrm{Lp}(\mathrm{a})$ is reported to be independent of age and gender ${ }^{2}$ but genetically linked to the isoform of the Kringle KIV-2, which exists in multiple copies ranging from 2 to $>40$. The size of the apo(a) isoform is inversely correlated to the plasma concentration of $\mathrm{Lp}$ (a) which explains up to $50 \%$ of the variability of the $\mathrm{Lp}(\mathrm{a})$ level in $\mathrm{man}^{3}$. Increasing plasma concentrations of $\mathrm{Lp}(\mathrm{a})$ are associated with the risk of cardiovascular disease $(\mathrm{CVD})^{4,5}$. Evidence for $\mathrm{Lp}(\mathrm{a})$ as a genetic risk factor for CVD is based on meta-analyses in 36 prospective studies $^{6}$, genetic studies ${ }^{7-9}$ and studies using the Mendelian randomization approach ${ }^{10,11}$.

In contrast to the clear positive association between $\mathrm{Lp}(\mathrm{a})$ and CVD risk a paradoxical inverse association of $\mathrm{Lp}$ (a) levels with risk for type 2 diabetes (T2DM) was published by Mora et al. ${ }^{12}$. This is counterintuitive as T2DM itself is a well known risk factor for CVD. In addition, Boronat et al..$^{13}$ identified a high level of Lp(a) at increased age to be protective for diabetes. Qi et al. ${ }^{14}$ reported that the $\mathrm{Lp}(\mathrm{a})$ genotype and $\mathrm{Lp}(\mathrm{a})$ plasma levels were not associated to CVD risk or CVD mortality in T2DM subjects in contrast to the general population. In vitro studies indicate that the synthesis of apo(a) is suppressed by insulin, suggesting that insulin has a secondary effect on $\mathrm{Lp}(\mathrm{a})$ and not vice vers ${ }^{15}$. Nevertheless, the role of $\mathrm{Lp}(\mathrm{a})$ in insulin resistance and glucose metabolism deserves further investigation to clarify the underlying mechanisms.

$\mathrm{Lp}$ (a) has similarities to both LDL and plasminogen and has therefore been proposed to possess proatherogenic properties, as well as prothrombotic properties ${ }^{16}$. The proposed proatherogenic mechanisms in vivo are numerous $^{17}$. It has been suggested that $\mathrm{Lp}(\mathrm{a})$ or the apo(a) molecule contributes to foam cell formation, smooth muscle cell (SMC) proliferation and migration, endothelial dysfunction and vascular inflammation, all known as part of the atheroma development ${ }^{18,19}$. The prothrombotic effect of $\mathrm{Lp}(\mathrm{a})$ is mediated by inhibition of plasminogen activation to plasmin, which is a result of competition between apo(a) and plasminogen for binding sites on fibrin ${ }^{20,21}$. However, apo(a), when bound to fibrin can per se bind and subsequently activate plasminogen to plasmin ${ }^{22}$ implying a more complex picture. 
Table 1 | Numbers and percentages of subjects in DIWA with different Lp(a) levels and diabetes status. Lp(a) levels are divided into quartiles. In the rightmost column, the total numbers and percentages of each subgroup (T2DM, IGT, NGT) are given. Means, medians and ranges for Lp(a) are given for each subgroup in the $1^{\text {st }}$ column

\begin{tabular}{lccccc}
$\begin{array}{l}\text { Diabetic status (mean, median, } \\
\text { range Lp(a)) }\end{array}$ & $\begin{array}{c}\text { 1 st Lp(a) quartile } \\
(<15.2 \mathrm{mg} / \mathrm{dL})\end{array}$ & $\begin{array}{c}\text { 2nd Lp(a) quartile } \\
(15.2-24.8 \mathrm{mg} / \mathrm{dL})\end{array}$ & $\begin{array}{c}\text { 3rd Lp(a) quartile } \\
(24.8-53.6 \mathrm{mg} / \mathrm{dL})\end{array}$ & $\begin{array}{c}\text { 4th Lp(a) quartile } \\
(>53.6 \mathrm{mg} / \mathrm{dL})\end{array}$ & All \\
\hline T2DM $(41.5 / 25.8 /[3.8,235.0])$ & $50(32.9 \%)$ & $57(37.3 \%)$ & $62(40.5 \%)$ & $56(36.4 \%)$ & $225(36.8 \%)$ \\
IGT $40.6 / 24.4 /[2.7,207.9])$ & $45(29.6 \%)$ & $57(37.3 \%)$ & $51(33.3 \%)$ & $52(33.8 \%)$ & $205(33.5 \%)$ \\
NGT $(40.5 / 22.2 /[0.9,200.8])$ & $57(37.5 \%)$ & $39(25.5 \%)$ & $40(26.1 \%)$ & $46(29.9 \%)$ & $182(29.7 \%)$ \\
\hline
\end{tabular}

An impaired fibrinolysis is described for diabetes subjects and has been linked to multiple alterations in coagulation/fibrinolysis pathways $^{23}$. Associations with high body mass index (BMI) and obesity are both linked to high plasminogen activator inhibitor 1 (PAI-1) plasma concentrations as well as high fibrinogen levels with effect on plasmin generation and fibrin clot structure. Furthermore, clots formed from purified fibrinogen from diabetic subjects showed a greater maximal absorbance at full polymerization than clots from control subjects ${ }^{24}$. Whether the maximal absorbance just reflects the average size of the fibrin fibers within the clot or the density of the fibrin network is still under discussion ${ }^{25,26}$.

The aim of this study was to contribute to the understanding of the role for $\mathrm{Lp}(\mathrm{a})$ in T2DM and hypofibrinolysis by an association study of Lp(a) plasma level to T2DM and plasma fibrinolysis in subjects from the previously described ${ }^{27}$ 'Diabetes and Impaired glucose tolerance in Women and Atherosclerosis study' (DIWA). Lp(a) measurements were made at an age of 64 (DIWA baseline) and reevaluated 5 years later (ReDIWA). The plasma clot lysis assay (CLA) was only performed on the ReDIWA material. To avoid the interference of PAI-1 and thrombin activatable fibrinolysis inhibitor (TAFI) with plasma fibrinolysis these two factors were blocked in the CLA.

\section{Results}

The baseline DIWA population $(n=612)$ with valid $\mathrm{Lp}(\mathrm{a})$ measures consist of 225 (36.8\%) T2DM subjects, 205 (33.5\%) subjects with impaired glucose tolerance (IGT) and $182(29.8 \%)$ with normal glucose tolerance (NGT) (Table 1), compared to the revisit population ReDIWA, $(n=465)$ with 203 T2DM subjects $(43.7 \%)$ and 262 NonT2DM (56.3\%) (Table 2).

A large variability in $\mathrm{Lp}(\mathrm{a})$ levels is seen in the baseline DIWA population, ranging from 2 to more than $200 \mathrm{mg} / \mathrm{dL}$ (Fig. 1a). The frequency distribution is highly skewed with approximately half of the population with an $\mathrm{Lp}$ (a) level below $25 \mathrm{mg} / \mathrm{dL}$, which can be seen in Table 1 where $\operatorname{Lp}(\mathrm{a})$ quartiles are shown. The three populations - T2DM, IGT and NGT - show a similar range of $\mathrm{Lp}(\mathrm{a})$ values and almost the same mean and median values (Table 1). In all four $\mathrm{Lp}$ (a) quartiles $33-40 \%$ of the subjects are diagnosed with T2DM and the dependence between diabetic status and $\operatorname{Lp}(\mathrm{a})$ was non-significant $(\mathrm{p}=0.30)$ using a chi-squared test.

The Lp(a) level in the T2DM subjects and NonT2DM in ReDIWA was similar to the baseline picture; there was a large variability in $\mathrm{Lp}$ (a) values for the diabetic and non-diabetic subjects and the distribution and mean value were similar for both groups (Fig. 1b). About $50 \%$ of the subjects in ReDIWA have an Lp(a) value below $10 \mathrm{mg} / \mathrm{dL}$, which is similar to the results from the Framingham Offspring population ${ }^{2}$. A cut-off level was set to $10 \mathrm{mg} / \mathrm{dL}$ (LOQ) which coincides with the median $\mathrm{Lp}(\mathrm{a})$ value (Table 2). The percentage of T2DM subjects was very similar in both groups, $43.0 \%$ in the lower $\mathrm{Lp}(\mathrm{a})$ group and $44.3 \%$ in the upper, and Fisher's exact test was clearly non-significant $(\mathrm{p}=0.96)$. Sixty-eight $(13.7 \%)$ of the NonT2DM subjects at baseline developed diabetes during the time to revisit, they were similar to the total population in regard to $\mathrm{Lp}(\mathrm{a})$ level and were equally distributed to the 4 baseline quartiles. Changes in $L p(a)$ level can be seen during these 5.5 years with an increase of more than $20 \%$ in $23 \%$ of the subjects and a decrease in $28 \%$, with no obvious relation to medication (statins, oral anti-diabetics, insulin, sex- and thyroid hormones).

Samples for the CLA were selected randomly in such way that the whole $\mathrm{Lp}$ (a) range was almost equally covered, even for higher $\mathrm{Lp}(\mathrm{a})$ levels where data are more sparse (Table 3). This means that almost all samples with an $\mathrm{Lp}$ (a) level $>40 \mathrm{mg} / \mathrm{dL}$ were analyzed, however a large proportion $(74.3 \%)$ of the ReDIWA samples with Lp(a) level $<10 \mathrm{mg} / \mathrm{dL}$ was not analyzed in CLA. This does not affect the percentage of T2DM subjects selected since the percentage with T2DM for those with $\mathrm{Lp}(\mathrm{a})<10 \mathrm{mg} / \mathrm{dL}$ was $43 \%$, which coincides very well with the numbers selected for CLA. Hence, also in this CLA population no correlation between the $\mathrm{Lp}$ (a) level and diabetes could be seen.

Clot lysis curves (Supplementary Fig. S1) have been obtained for 274 subjects and different clot lysis parameters were calculated by the appropriate software: clot lysis time (CLT), maximal Absorption at $405 \mathrm{~nm}\left(\mathrm{~A}_{\max }\right)$ and the area under the clot lysis curve (AUC).

The CLT in T2DM was significantly higher than in NonT2DM subjects (Fig. 2a), while the CLT is similar in the $4 \mathrm{Lp}$ (a) quartiles. A large proportion of the subjects (67\%) have a CLT between 10000 and 30000 seconds. The frequency distribution is highly skewed with CLT up to $70000 \mathrm{~s}$. The maximal absorbance $\left(\mathrm{A}_{\max }\right)$ was increased with increasing $\mathrm{Lp}(\mathrm{a})$ level for both populations in a similar fashion (Fig. 2b). The distribution is close to normal with a mean of $1 \mathrm{OD}$ ranging from 0.3 to 1.6 units. As a result of these two findings, the AUC of the clot lysis curve is significantly dependent on the diabetes status and on the Lp(a) plasma concentration (Fig. 2c). The AUC increases with increasing CLT as seen in the T2DM subjects, an Lp(a) dependent increase in $A_{\max }$ increases the AUC as well.

Comparing the CLA readings for the subjects with $\operatorname{Lp}(\mathrm{a})$ level in the $2^{\text {nd }}, 3^{\text {rd }}$ and $4^{\text {th }}$ quartile and those subjects in the $1^{\text {st }} \mathrm{Lp}(\mathrm{a})$ quartile (Table 4) the above described situation is underpinned with relevant p-values. For the CLT there is no difference between the $1^{\text {st }} \mathrm{Lp}(\mathrm{a})$ quartiles and all others, while for $A_{\max }$ and AUC, subjects within the $1^{\text {st }} \mathrm{Lp}(\mathrm{a})$ quartile are significantly different from those in the $3^{\text {rd }}$ and $4^{\text {th }}$ quartile. Even in relation to the diabetes status the CLA results reach a high level of significance (Table 5) with an estimated difference of $35.4 \%$ for CLT and $29.4 \%$ for AUC while there is no difference between T2DM and NonT2DM for $A_{\text {max }}$.

For 628 of the 633 women included from start, there is information available regarding the incidence of death, myocardial infarction or stroke (MACE) at the end of 2008. The total event rate for these endpoints was $7.5 \%$ (47 events). Only three of the events occurred in

Table 2 | Numbers and percentages of subjects in ReDIWA with different $L p(a)$ levels and diabetes status. $L p(a)$ levels are divided into two groups. In the rightmost column, the total numbers and percentages of the two subgroups (T2DM and NonT2DM) are given

\begin{tabular}{lccc} 
& $\begin{array}{c}\text { Lower half } \\
(<\mathbf{1 0} \mathbf{~ m g / d L})\end{array}$ & $\begin{array}{c}\text { Upper half } \\
(>\mathbf{1 0} \mathbf{~ m g / d L})\end{array}$ & All \\
\hline T2DM & $101(43 \%)$ & $102(44.3 \%)$ & $203(43.7 \%)$ \\
NonT2DM & $134(57 \%)$ & $128(55.7 \%)$ & $262(56.3 \%)$ \\
\hline
\end{tabular}


(a)

Lp(a), DIWA

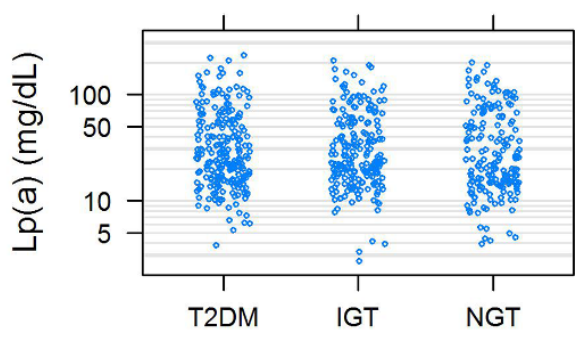

050150250

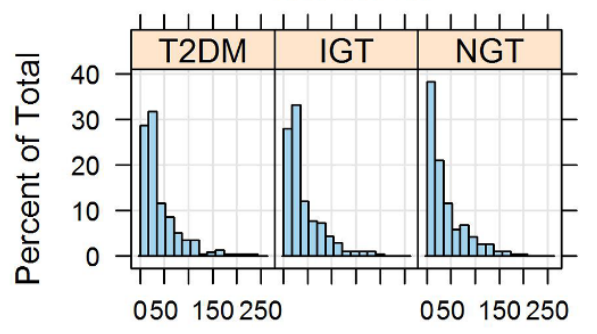

$\mathrm{Lp}(\mathrm{a})(\mathrm{mg} / \mathrm{dL})$ (b)

Lp(a), ReDIWA
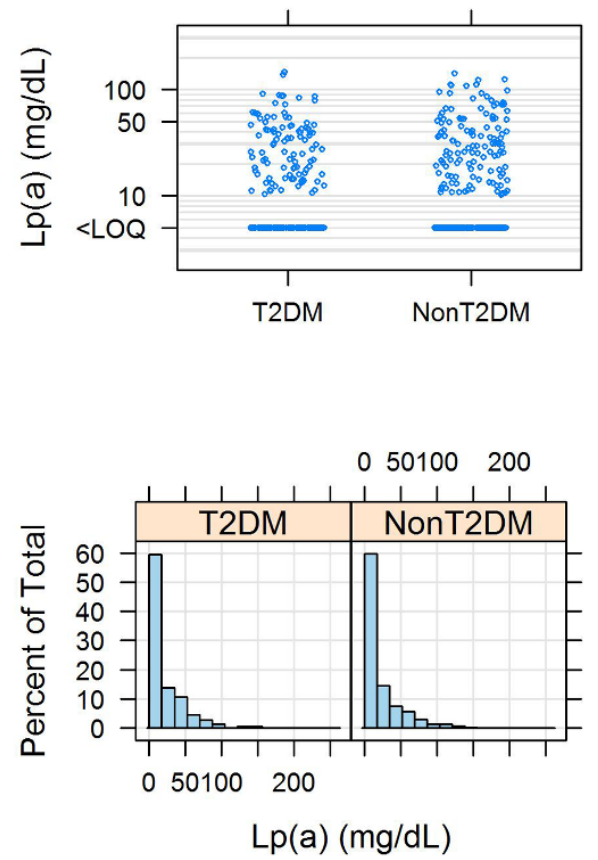

Figure $1 \mid \mathrm{Lp}$ (a) plasma levels and distribution vs. diabetes status in DIWA and ReDIWA. Distribution of Lp(a) plasma level in (a) DIWA 64 year old women with T2DM $(n=225)$, impaired glucose tolerance (IGT) $(n=205)$ and controls with normal glucose tolerance (NGT) $(n=182)$ and in $(\mathrm{b})$ ReDIWA 69 year old women with T2DM $(n=203)$ or nonT2DM $(n=262)$. Lp(a) level s are shown in $\mathrm{mg} / \mathrm{dL}$ (upper panel) on a log scale and as histogram (lower panel).

the non-diabetic group (baseline assessment) which means that there is a significant difference between diabetic and non-diabetic women regarding event rate. In terms of odds ratios the risk of event is 6.3 in the T2DM group vs. NonT2DM ( $<<0.001, \mathrm{CI}:(3.3,13.4))$. Lp(a) however, which was available for 612 of these 628 women, is not observed to be related to the occurrence of events; the percentages were $9.9 \%, 7.2 \%, 3.3 \%$ and $9.7 \%$ in the different quartiles, starting with the lowest one. No interaction term was included since this did not improve the model fit.

\section{Discussion}

In this study no relationship between $\mathrm{Lp}(\mathrm{a})$ and T2DM could be seen, which is in contrast to the publications mentioned in the introduction. Recently, the inverse association between $\mathrm{Lp}(\mathrm{a})$ and risk for $\mathrm{T}_{2} \mathrm{DM}^{12}$ has been modified by Kamstrup and Nordestgaard at the 81. EAS Congress $2013^{28}$. The genetic variance of the Kringle KIV-2 is related to type 2 diabetes risk, whereas low Lp(a) levels per se are not. Their findings imply that the genetically determined structure of $\mathrm{Lp}(\mathrm{a})$, i.e. the LPA KIV-2 genotype, causes the association of $\mathrm{Lp}(\mathrm{a})$ with increased risk of diabetes. In earlier studies the LPA KIV-2 genotype was shown to explain approximately $50 \%$ of the variability of plasma $L p(a)$ levels ${ }^{1,3,6}$. However, recent more extended analysis

Table $3 \mid$ Number of individuals in the different $L p(a)$ ranges in ReDIWA, selected for Clot lysis assay. The percentage of T2DM patients in these numbers are given in parenthesis

\begin{tabular}{lrc} 
Lp(a) range & ReDIWA & CLA cohort \\
\hline $\operatorname{Lp}(a)<10$ & $237(43 \%)$ & $61(44 \%)$ \\
$10<\operatorname{Lp}(a) \leq 20.93$ & $79(47 \%)$ & $69(48 \%)$ \\
$20.93<\operatorname{Lp}(a) \leq 41.97$ & $79(42 \%)$ & $67(42 \%)$ \\
$\operatorname{Lp}(a)>41.97$ & $79(41 \%)$ & $77(40 \%)$ \\
\hline
\end{tabular}

came to the conclusion that the KIV-2 genotype explains only 21$27 \%$ of the variability of $L p(a)$ plasma levels, dependent on the cohort analyzed $^{29}$. Our findings support this recent modification of the relationship between $\mathrm{Lp}(\mathrm{a})$ and T2DM.

An additional explanation for the different views in the literature is that $\mathrm{Lp}(\mathrm{a})$ is challenging to measure ${ }^{30}$. In interventional studies, e.g. statin trials, where $\mathrm{Lp}(\mathrm{a})$ was included in the lipoprotein profile, all samples were analyzed in one batch at the end of the study to avoid batch to batch variability and freeze thaw cycles. Kronenberg et al. described a decrease in $\mathrm{Lp}(\mathrm{a})$ concentrations in EDTA plasma of approximately $10 \%$ during the first month of storage at $-80^{\circ} \mathrm{C}^{31}$. Simó et al. ${ }^{32}$ suggested that Lp(a) in EDTA plasma decreased significantly in MI survivors compared to healthy controls when stored at $-70^{\circ} \mathrm{C}$ for up to 5 years, despite the addition of antioxidants and proteolysis inhibitors. However this was only observed in samples with Lp(a) concentrations above $40 \mathrm{mg} / \mathrm{dl}$. Also in the present study we struggled with different matrices and methods and different storage periods at $-80^{\circ} \mathrm{C}$. Nevertheless an effort was made to compare the $\operatorname{Lp}(\mathrm{a})$ values at baseline with the re-examination values assessed for the same patient. Almost half of the subjects had changes in $\mathrm{Lp}(\mathrm{a})$ that were above $20 \%$, with a CV\% for both Lp(a) assays below $10 \%$. In contrary to results described by Simó ${ }^{32}$ this was not related to the baseline Lp(a) plasma concentrations and was not different in diabetes compared to non-diabetic subjects. Inconsistencies in $\mathrm{Lp}(\mathrm{a})$ measures during longitudinal studies can be related to the poor standardization of methodology and the lack of reference material ${ }^{30}$. The reason for the variability in $\operatorname{Lp}(\mathrm{a})$ over time may be related to individual variations in the $\mathrm{Lp}(\mathrm{a})$ particle size $\mathrm{e}^{33}$, and variability in the metabolism of $\mathrm{Lp}(\mathrm{a})$ which is largely dependent on liver and kidney function. However, the $\mathrm{Lp}$ (a) levels in this study were comparable to those published from the large Copenhagen Heart study'.

Our study population was selected with regard to age and gender the women were 64 years at inclusion and 69 years at follow-up and 


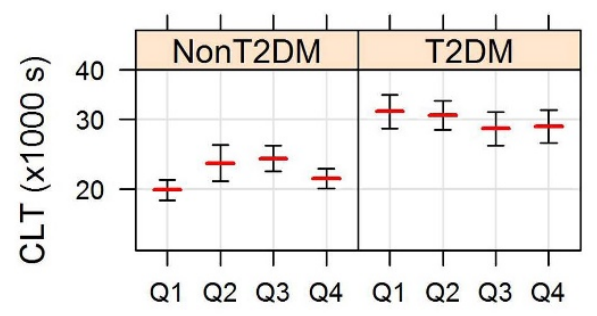

$\mathrm{Lp}(\mathrm{a})$ quartile

(b) $A_{\max }$ vs $L p(a)$ for NonT2DM / T2DM

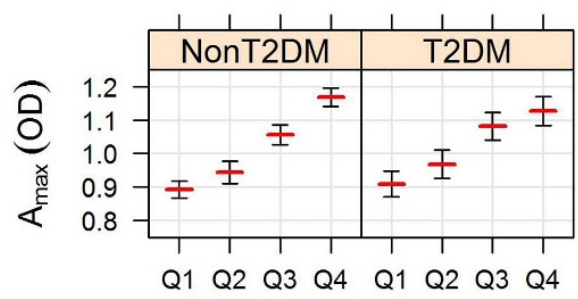

$L p(a)$ quartile (c) AUC vs Lp(a), for NonT2DM / T2DM

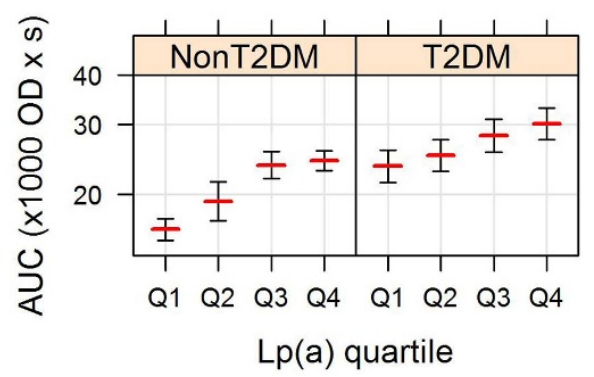

Figure $2 \mid$ Clot lysis assay results in relation to $\mathrm{Lp}(\mathrm{a})$ plasma levels and diabetes status. The CLA results for the CLA population of 69 year old women $(n=265)$ grouped for the $\operatorname{Lp}(\mathrm{a})$ quartiles and diabetes status. The CLA variables are shown as mean values $\pm \operatorname{SEM}$ in the four Lp(a) quartiles. (a) Clot lysis time (CLT) [s]. (b) Maximal Peak height (Amax) [OD]. (c) Area under the clot lysis curve (AUC) [OD $\times$ s].

the results may not be extended beyond this cohort. However, focusing on this cohort can be seen as a strength, since confounding factors such as age, sex, ethnicity and premenopausal variability are ruled out $^{34}$. A significant association of elevated Lp(a) level and CVD in subjects above 55 years of age was reported by $\mathrm{Balogh}^{35}$; and was more pronounced in women than in men. The same association between age and $\mathrm{Lp}(\mathrm{a})$ levels in women was reported by Jenner ${ }^{2}$, who analyzed the Lp(a) levels in the Framingham offspring study population. The mean plasma concentration in postmenopausal women was $19 \%$ higher than the mean found in premenopausal subjects.

This study also shows that T2DM patients have decreased fibrinolysis, also when PAI-1 and TAFIa are blocked. The increased thrombosis risk in subjects with diabetes is in part due to decreased fibrinolytic potential as measured by the $\mathrm{CLA}^{36}$. This is in line with Dunn's results on fibrin clots formed from fibrinogen obtained from subjects with $\mathrm{T} 2 \mathrm{DM}^{37}$. Such tightly packed clots with a denser less

Table 4 | Clot lysis assay results in relation to Lp(a) plasma levels. Comparisons of the main CLA read outs between subjects of the CLA cohort in the 2nd, 3rd and 4th Lp(a) quartile and subjects in the 1 st Lp(a) quartile

\begin{tabular}{|c|c|c|c|}
\hline Variable & Comparison & $\begin{array}{l}\text { Estimated difference } \\
(95 \% \mathrm{CI})\end{array}$ & P-value \\
\hline \multirow{3}{*}{$A_{\max }$} & $2^{\text {nd }}-1^{\text {st }}$ & $0.06 \mathrm{OD}(-0.02,0.14)$ & 0.238 \\
\hline & $3^{\text {rd }}-1^{\text {st }}$ & 0.17 OD $(0.09,0.25)$ & $<0.001$ \\
\hline & $4^{\text {th }}-1^{\text {st }}$ & $0.25 \mathrm{OD}(0.17,0.33)$ & $<0.001$ \\
\hline \multirow[t]{3}{*}{ CLT } & $2^{\text {nd }} \mathrm{cf} 1^{\text {st }}$ & $8.3 \%(-10.8,31.6)$ & 0.645 \\
\hline & $3^{\text {rd }} \mathrm{cf}^{\text {st }}$ & $6.6 \%(-12.4,29.8)$ & 0.782 \\
\hline & $4^{\text {th }}$ cf $1^{\text {st }}$ & $0.4 \%(-17.5,22.1)$ & $>0.999$ \\
\hline \multirow[t]{3}{*}{ AUC } & $2^{\text {nd }} \mathrm{cf}^{\text {st }}$ & $1.13 \%(0.93,1.38)$ & 0.333 \\
\hline & $3^{\text {rd }} \mathrm{cf}^{\text {st }}$ & $1.34 \%(1.10,1.64)$ & 0.002 \\
\hline & $4^{\text {th }}$ cf $1^{\text {st }}$ & $1.40 \%(1.15,1.71)$ & $<0.001$ \\
\hline
\end{tabular}

porous structure are more resistant to fibrinolysis than those from healthy subjects. Increased glycosylation of fibrinogen caused by hyperglycemia has been related to this clot structure type ${ }^{38}$.

Furthermore, fibrinogen is known to be increased in T2DM, more pronounced in women in parallel with CRP and mirrors the inflammatory status in these subjects. Even in this study a positive correlation between $A_{\max }$ and CRP plasma levels was seen. Recent CLA results from the Edinburgh Type 2 Diabetes study ${ }^{39}$ show a gender specific alteration in clot structure. A higher maximum absorbance and longer clot lysis times are reported for women with T2DM compared to male T2DM subjects. Laser scanning confocal microscopy showed a denser ex vivo fibrin clot for T2DM women. We used the patient's own ability to generate thrombin in plasma and blocked the fibrinolysis inhibitors PAI- 1 and TAFI. This system has been established in our laboratory with an antibody to PAI-1 (Fab fragment of MAI-12) that binds specifically to active PAI-1 at a concentration sufficient even for the high PAI-1 plasma activities that are described for diabetes. This antibody has been used in vivo in a rat model of arterial thrombosis and caused a reduction in thrombus size and restored blood flow ${ }^{40}$. TAFI is blocked by a compound (AZD 9684, AstraZeneca R\&D) that specifically inhibits active TAFI (TAFIa) and hence increases fibrinolysis in the plasma clot lysis assay already at low concentrations $\left(\mathrm{IC}_{50}=0.35 \mu \mathrm{mol} / \mathrm{L}\right)$. Differences in methodology with regard to the initiation of clotting and blockage of PAI-1 and TAFIa may explain the difference between studies.

However, in the present study Lp(a) plasma levels had no effect on clot-lysis time but increased $\mathrm{Lp}(\mathrm{a})$ levels caused an increase in $\mathrm{A}_{\text {max }}$. Undas et al. ${ }^{41}$ describe a longer fibrinolysis time associated with increased $L p(a)$ level in a similar clot lysis assay. They explain the fibrinolysis impairment by $\mathrm{Lp}(\mathrm{a})$ interference with plasminogen binding to fibrin but also show data on $\mathrm{Lp}(\mathrm{a})$ induced alteration in fibrin clot structure that suggest a prothrombotic effect of $\operatorname{Lp}(a)^{42}$.

The maximal absorption $A_{\max }$ of a clot lysis curve is known to be dependent on the initial thrombin generation and the fibrinogen level in plasma, since both factors determine the development of 
Table 5 | Clot lysis assay results in relation to diabetes status. Comparisons of the main CLA read outs between subjects with T2DM and nonT2DM

\begin{tabular}{lccr} 
Variable & Comparison & $\begin{array}{c}\text { Estimated } \\
\text { difference }(\mathbf{9 5} \% \mathbf{C I})\end{array}$ & P-value \\
\hline $\mathrm{A}_{\max }$ & $\mathrm{DM}-\mathrm{no} \mathrm{DM}$ & $-0.007 \mathrm{OD}(-0.055,0.042)$ & 0.787 \\
$\mathrm{CLT}$ & $\mathrm{DM} \mathrm{cf} \mathrm{no} \mathrm{DM}$ & $35.4 \%(20.5,52.2)$ & $<0.001$ \\
$\mathrm{AUC}$ & $\mathrm{DM}$ cf no DM & $29.4 \%(14.8,45.8)$ & $<0.001$ \\
\hline
\end{tabular}

fibrin protofibriles that determine the fibrin clot structure ${ }^{37}$. We show that $L p(a)$ influences the clot structure but the mechanism is not obvious. One possibility is that $\mathrm{Lp}(\mathrm{a})$ is influencing thrombin generation during re-calcification. A second possibility is that $\mathrm{Lp}(\mathrm{a})$ binds to the fibrin structure, without inhibiting fibrinolysis. This may increase the clot mass or influence clot structure and result in an increase in $A_{\max }$. Altered fibrin clot properties represent a novel prothrombotic effect of $\mathrm{Lp}(\mathrm{a})$ which is of potential importance in athero-thrombotic vascular disease $\mathrm{e}^{43,44}$. $\mathrm{Lp}$ (a) seems to be one of the factors that contribute to changes in fibrin clot structure which may in vivo affect plaque stability under flow conditions in arteriosclerotic vessels. Beyond this $\operatorname{Lp}(\mathrm{a})$ is described to alter plasmin generation on the clot surface and to inhibit plasminogen binding to endothelial cells affecting t-PA release. Both mechanisms may contribute to the development of a more rigid clot and by this increase the risk for CVD. Further studies will have to study these findings in greater detail.

In conclusion no difference in $\mathrm{Lp}(\mathrm{a})$ levels between T2DM and NonT2DM could be seen in this study. Diabetes subjects show impaired fibrinolysis potential, due to a prolonged clot lysis time, that was not related to PAI-1, TAFI or Lp(a). Lp(a) levels are related to an increase in the maximal absorbance $\mathrm{A}_{\max }$.

\section{Methods}

Study cohort. The Diabetes and Impaired glucose tolerance in Women and Atherosclerosis study (DIWA) is a population-based cohort of 64-year-old women who participated in a screening examination, as previously described in detail ${ }^{27}$. World Health Organization (WHO) criteria were used for the definitions of diabetes mellitus and impaired glucose tolerance (IGT) ${ }^{45}$. Women with diabetes and similarly sized groups of women with IGT and normal glucose tolerance (NGT) were randomly selected and invited to participate in a nested case-control study. In total, 633 women participated (diabetes, $n=234$; IGT, $n=208$; NGT, $n=191$ ) in an oral glucose tolerance test (OGTT) and underwent ultrasound examination of the carotid arteries and clinical and laboratory examinations.

The baseline examination (DIWA) included a questionnaire regarding previous diseases, current medication, smoking habits, alcohol intake, physical activity and family history of diabetes. Anthropometric measurements, blood pressure and heart rate were recorded. Ultrasound examination of the carotid arteries was performed, blood samples were collected, and serum and plasma were frozen in aliquots at $-80^{\circ} \mathrm{C}$ within $4 \mathrm{~h}$. Clinical chemistry laboratory analyses of plasma for lipoproteins and inflammatory and diabetes parameters were performed as previously described ${ }^{46,47}$.

A re-examination (ReDIWA) was performed after 5 to 6 years (mean 5.5 years) ${ }^{48}$. At re-examination, subjects underwent similar investigations to those performed at baseline. OGTT was performed in subjects who were NonT2DM at baseline in order to examine whether subjects developed diabetes during the period between baseline and follow-up. Subjects who had available plasma samples $(n=465), 203(43.9 \%)$ T2DM subjects and 262 (56.1\%) NonT2DM, were investigated for Lp(a).

Cardiovascular events and cause of death were investigated by contact with the centre of Epidemiology at the national Board of Health and Welfare. All subjects received both written and oral information before providing consent to participate in the study. The protocol was approved by the Ethics Committee at Sahlgrenska University Hospital, Gothenburg Sweden. All experiments were performed in accordance with the relevant guidelines and regulations.

Lipoprotein(a) analysis. Blood from DIWA and ReDIWA subjects was taken by venipuncture and serum and citrate plasma was harvested after centrifugation at $1500 \mathrm{~g}$ for $20 \mathrm{~min}$ at $4^{\circ} \mathrm{C}$. Supernatants were stored as $0.5 \mathrm{ml}$ portions at $-80^{\circ} \mathrm{C}$ until analysis.

Analytical methods for $\operatorname{Lp}(\mathrm{a})$ are all based on the apo(a) molecule that characterizes this lipoprotein particle and a large variety of antibodies to apo(a) can be found, due to the polymorphic nature of this highly glucosylated protein. Currently antibodies are available that do not recognize the Kringle IV-2 repeat and thus allow a size independent quantification of $\mathrm{Lp}(\mathrm{a})$. Immunochemical techniques like immunoturbidimetry (ITM) or ELISA are the methods of choice in today's clinical chemical laboratory.

Serum has been subjected to ITM for the baseline samples that had been stored at $-80^{\circ} \mathrm{C}$ for more than a year, dependent on the inclusion period of the study. Citrate plasma was the matrix for the ELISA used for the ReDIWA samples, which had also been stored at $-80^{\circ} \mathrm{C}$ for 2 to 4 years. The turbidimetric method has a higher sensitivity while the LOQ for the ELISA is $10 \mathrm{mg} / \mathrm{dL}$. Samples of the 465 subjects investigated in ReDIWA were analyzed for Lp(a) by ELISA Kamiya (Kamiya Biomedical Company, Seattle, USA) on an ABX pentra analyzer and the results compared to the baseline data obtained by immuno-turbidimetry with the same Kamiya kit on the Konelab 20 machine.

Due to differences in $\mathrm{Lp}(\mathrm{a})$ assay and matrix for the analysis in DIWA and ReDIWA there was a discrepancy between these two data sets. The baseline Lp(a) concentrations were in general higher than the ReDIWA values. In an attempt to compare the baseline and revisit Lp(a) levels for each subject, Deming regression of revisit vs. baseline values was used ${ }^{49}$. Using the achieved regression equation, the revisit $\mathrm{Lp}(\mathrm{a})$ values were adjusted to be comparable to the baseline values (Supplementary Fig. S2).

Clot lysis assay (CLA). The CLA method is based on the registration of fibrin aggregation in human plasma and describes the kinetics of fibrin clot lysis ex vivo. The assay has been widely used and modified to evaluate the effect of different coagulation/fibrinolysis plasma proteins on this process ${ }^{50,51}$. Common modifications of this method include coagulation activation by Tissue factor (TF) and addition of thrombin to enhance the clotting process ${ }^{41}$. We use the patient's own coagulation properties and initiate clotting by recalcification of the citrated plasma. Tissueplasminogen activator ( $t$-PA) was added at low concentration to support plasminogen activation to plasmin.

Plasmin is the main fibrinolytic enzyme which is inhibited at different levels. Alpha 2-Antiplasmin inhibits the enzyme activity directly, while plasminogen activator inhibitor-1 (PAI-1) neutralizes t-PA and prevents plasminogen activation. Thrombin activatable fibrinolysis inhibitor (TAFI) interferes with plasminogen binding to fibrin by cleavage of $\mathrm{C}$-terminal lysine residues, the main anchor sites on partially degraded clots. Since apo(a) competes with plasminogen for these binding sites, an inhibitory effect of $\mathrm{Lp}(\mathrm{a})$ on clot lysis is discussed ${ }^{19}$. To circumvent the confounding effects we inhibited the two major players PAI-1 and TAFI in the clot lysis assay ${ }^{52}$. The monoclonal antibody MAI-12 inhibits t-PA binding to PAI- $1^{53}$. TAFI is inhibited by a compound developed by AstraZeneca ${ }^{54}$ specifically inhibiting the enzymatic activity. The clot lysis curves obtained with this modification (Supplementary Fig. S1) are similar to published examples ${ }^{50}$. In a first attempt to measure CLA without inhibition of PAI-1 and TAFI, the variability was so large that no trends could be seen.

Citrate plasma from the CLA cohort was rapidly thawed in a water bath to a temperature of $37^{\circ} \mathrm{C}$ in parallel with all other constituents, except for t-PA. 96 well flat bottom microtitre plates (Nunc, Denmark; 269629) were loaded with $25 \mu \mathrm{l} \mathrm{CaCl}_{2}$ (KEBO, Sweden) in $27 \mathrm{mM}$ Hepes pH7.4, $20 \mathrm{mM} \mathrm{NaCl}$ ), $25 \mu \mathrm{lMAI}-12$ (Fab Biopool, Sweden) and $25 \mu \mathrm{l}$ AZD9684. $100 \mu \mathrm{l}$ plasma and $25 \mu \mathrm{l}$ cold two-chain tissue plasminogen activator ( $t$-PA, Biopool, Sweden in saline with $0.3 \%$ Tween 80 ) were mixed and added into the wells resulting in final concentrations of $5 \mathrm{ng} / \mathrm{mL}$ t-PA, $1 \mu \mathrm{M}$ MAI-12, $10 \mu \mathrm{M}$ AZD9684 and $7.5 \mathrm{mM} \mathrm{CaCL}_{2}$.

The lid covered plate was placed in a Thermomax microplate reader (Molecular Devices, US) and gently shaken. The change in turbidity was monitored as a change in absorbance at $405 \mathrm{~nm}$ at $37^{\circ} \mathrm{C}$ for up to $10 \mathrm{~h}$ with repetitive readings each second minute. From the resulting clot lysis curve different variables were calculated using the reader software SoftMax Pro 4.8 (Molecular Devices, US). The maximal Absorbance $\left(\mathrm{A}_{\max }\right)$ is given by the difference between the highest absorption at OD 405 and the baseline absorption (Supplementary Fig. S1). Clot lysis time (CLT) is defined as the time from maximal clotting velocity to maximal lysis velocity and is similar to the peak width (sec.) at half maximal absorption $\left(A_{\max } / 2\right)$, even called $t_{50 \%}$ or $T_{1 / 2}$ by others ${ }^{26,55}$. Furthermore the area under the clot lysis curve (AUC) is calculated, which is dependent on $\mathrm{A}_{\max }$ as well as on the CLT.

Statistical analysis. The statistical analysis and graphs were performed using R version 2.13.2.

The dependence between $\operatorname{Lp}(\mathrm{a})$ and diabetic status was tested both at baseline and revisit. Since the assay used for $\mathrm{Lp}(\mathrm{a})$ at revisit had a lower sensitivity, which contributed to half of the samples being below LOQ $=10 \mathrm{mg} / \mathrm{dL}$, these $\mathrm{Lp}$ (a) levels were divided into two groups, above and below LOQ. Regarding diabetic status, two levels were available at revisit; T2DM and NonT2DM. Fisher's exact test was used to test for independence. At baseline, Lp(a) measurements were divided into quartiles and there were three groups for diabetic status: T2DM, IGT and NGT. Here a chi-squared test was used to test independence owing to the size of the data set.

To test whether diabetic status and Lp(a) are of importance for the clot lysis, twoway analyses of variance (ANOVA) were applied. For CLT and AUC the data were approximately log normally distributed and therefore log transformed before the analysis, while $A_{\max }$ was analyzed on the original scale. This is the reason that the results for CLT and AUC are presented as percentage change, while for $A_{\max }$ the difference between groups is presented in the same scale as the data. No interaction terms were included since their inclusion did not improve the model fits (testing model improvement by means of chi-square tests gives $\mathrm{p}>0.4$ in all three cases). The $\mathrm{Lp}$ (a) values for the subjects included in the CLA were divided into quartiles, for the first quartile the major part of the data were below LOQ. For each of CLT, AUC and 
$A_{\max }$, Dunnett's test, which adjusts for multiple comparisons, was performed to compare the three upper $\operatorname{Lp}(\mathrm{a})$ quartiles with the lower one.

To investigate the effect of diabetes status and Lp(a) levels at baseline on the event rate MACE, a logistic regression model was used. An interaction term again did not improve the model fit and hence was not included in the final model. Due to its right skewed distribution, $\mathrm{Lp}$ (a) levels were log transformed. The results are presented in terms of odds ratios which describe how strongly diabetes and $\mathrm{Lp}(\mathrm{a})$ levels are associated with event rate.

Due to differences in the $\mathrm{Lp}(\mathrm{a})$ assays and matrix for the analysis in DIWA and ReDIWA there is a discrepancy between these two data sets. A Deming regression, which takes into account the variability in both revisit and baseline values, was fitted to the $\mathrm{Lp}(\mathrm{a})$ data on the log scale. To be able to identify subjects whom had changed more than $20 \%$ from start to revisit, the $\mathrm{Lp}$ (a) values at revisit were adjusted by means of the fitted equation. Only subjects who had Lp(a) values at revisit above LOQ were included in this analysis.

1. Kronenberg, F. \& Utermann, G. Lipoprotein(a): resurrected by genetics. J. Intern. Med. 273, 6-30 (2013).

2. Jenner, J. L. et al. Effects of age, sex, and menopausal status on plasma lipoprotein(a) levels. The Framingham Offspring Study. Circulation 87, 1135-1141 (1993).

3. Nordestgaard, B. G. \& Tybjærg-Hansen, A. Genetic determinants of LDL, lipoprotein(a), triglyceride-rich lipoproteins and HDL: concordance and discordance with cardiovascular disease risk. Curr. Opin. Lipidol. 22, (2011).

4. Tsimikas, S. \& Hall, J. L. Lipoprotein(a) as a Potential Causal Genetic Risk Factor of Cardiovascular Disease. J. Am. Coll. Cardiol. 60, 716-721 (2012).

5. Enkhmaa, B., Anuurad, E., Zhang, W., Tran, T. \& Berglund, L. Lipoprotein(a): genotype-phenotype relationship and impact on atherogenic risk. Metab. Syndr. Relat. Disord. 9, 411-418 (2011).

6. Erqou, S. et al. Lipoprotein(a) concentration and the risk of coronary heart disease, stroke, and nonvascular mortality. JAMA - Journal of the American Medical Association 302, 412-423 (2009).

7. Helgadottir, A. et al. Apolipoprotein(a) genetic sequence variants associated with systemic atherosclerosis and coronary atherosclerotic burden but not with venous thromboembolism. J. Am. Coll. Cardiol. 60, 722-729 (2012).

8. Clarke, R. et al. Genetic variants associated with $\mathrm{Lp}(\mathrm{a})$ lipoprotein level and coronary disease. N. Engl. J. Med. 361, 2518-2528 (2009).

9. Kamstrup, P. R., Tybjærg-Hansen, A., Steffensen, R. \& Nordestgaard, B. G. Genetically elevated lipoprotein(a) and increased risk of myocardial infarction. JAMA - Journal of the American Medical Association 301, 2331-2339 (2009).

10. Kamstrup, P. R., Tybjærg--Hansen, A. \& Nordestgaard, B. G. Genetic Evidence That Lipoprotein(a) Associates With Atherosclerotic Stenosis Rather Than Venous Thrombosis. Arteriosclerosis, Thrombosis, and Vascular Biology 32, 1732-1741 (2012).

11. Thanassoulis, G. et al. Genetic Associations with Valvular Calcification and Aortic Stenosis. N. Engl. J. Med. 368, 503-512 (2013).

12. Mora, S. et al. Lipoprotein(a) and risk of type 2 diabetes. Clin. Chem. 56 1252-1260 (2010)

13. Boronat, M. et al. High levels of lipoprotein(a) are associated with a lower prevalence of diabetes with advancing age: Results of a cross-sectional epidemiological survey in Gran Canaria, Spain. Cardiovascular Diabetology 11, 81 (2012)

14. Qi, Q., Workalemahu, T., Zhang, C., Hu, F. B. \& Qi, L. Genetic variants, plasma lipoprotein(a) levels, and risk of cardiovascular morbidity and mortality among two prospective cohorts of type 2 diabetes. European Heart Journal 33, 325-334 (2012).

15. Neele, D. M., de Wit, E. C. \& Princen, H. M. Insulin suppresses apolipoprotein(a) synthesis by primary cultures of cynomolgus monkey hepatocytes. Diabetologia 42, 41-44 (1999).

16. Koschinsky, M. L. Novel insights into Lp(a) physiology and pathogenicity: more questions than answers? Cardiovasc. Hematol. Disord. Drug Targets 6, 267-278 (2006).

17. Nordestgaard, B. G. et al. Lipoprotein(a) as a cardiovascular risk factor: current status. Eur. Heart J. 31, 2844-2853 (2010).

18. Anuurad, E., Boffa, M. B., Koschinsky, M. L. \& Berglund, L. Lipoprotein(a): a unique risk factor for cardiovascular disease. Clin. Lab. Med. 26, 751-772 (2006).

19. Feric, N. T., Boffa, M. B., Johnston, S. M. \& Koschinsky, M. L. Apolipoprotein(a) inhibits the conversion of Glu-plasminogen to Lys-plasminogen: a novel mechanism for lipoprotein(a)-mediated inhibition of plasminogen activation. J. Thromb. Haemost. 6, 2113-2120 (2008).

20. Angles-Cano, E., de la Pena Diaz, A. \& Loyau, S. Inhibition of fibrinolysis by lipoprotein(a). Ann. N. Y. Acad. Sci. 936, 261-275 (2001).

21. Hancock, M. A., Boffa, M. B., Marcovina, S. M., Nesheim, M. E. \& Koschinsky, M. L. Inhibition of plasminogen activation by lipoprotein(a): critical domains in apolipoprotein(a) and mechanism of inhibition on fibrin and degraded fibrin surfaces. J. Biol. Chem. 278, 23260-23269 (2003).

22. Liu, J., Harpel, P. C. \& Gurewich, V. Fibrin-bound lipoprotein(a) promotes plasminogen binding but inhibits fibrin degradation by plasmin. Biochemistry 33, 2554-2560 (1994)

23. Alzahrani, S. H. \& Ajjan, R. A. Coagulation and fibrinolysis in diabetes. Diab Vasc. Dis. Res. 7, 260-273 (2010).
24. Dunn, E. J., Philippou, H., Ariens, R. A. \& Grant, P. J. Molecular mechanisms involved in the resistance of fibrin to clot lysis by plasmin in subjects with type 2 diabetes mellitus. Diabetologia 49, 1071-1080 (2006).

25. Collet, J. P. et al. Altered fibrin architecture is associated with hypofibrinolysis and premature coronary atherothrombosis. Arterioscler. Thromb. Vasc. Biol. 26 2567-2573 (2006).

26. Bochenek, M., Zalewski, J., Sadowski, J. \& Undas, A. Type 2 diabetes as a modifier of fibrin clot properties in patients with coronary artery disease. J. Thromb. Thrombolysis 35, 264-270 (2013).

27. Brohall, G., Behre, C. J., Hulthe, J., Wikstrand, J. \& Fagerberg, B. Prevalence of diabetes and impaired glucose tolerance in 64-year-old Swedish women: experiences of using repeated oral glucose tolerance tests. Diabetes Care 29 363-367 (2006)

28. Kamstrup, P. R. \& Nordestgaard, B. G. Lipoprotein(a) is causally associated with type 2 diabetes. 81st EAS Congress (2013) (Date of access 11 April 2014) http://w3. kenes-group.com/apps/eas2013/abstracts/pdf/1177.pdf.

29. Kamstrup, P. R., Tybjaerg-Hansen, A. \& Nordestgaard, B. G. Lipoprotein(a) and risk of myocardial infarction--genetic epidemiologic evidence of causality. Scand. J. Clin. Lab. Invest. 71, 87-93 (2011).

30. Kostner, K. M., Marz, W. \& Kostner, G. M. When should we measure lipoprotein (a)? Eur. Heart J. 34, 3268-3276 (2013).

31. Kronenberg, F., Lobentanz, E. M., Konig, P., Utermann, G. \& Dieplinger, H. Effect of sample storage on the measurement of lipoprotein[a], apolipoproteins B and AIV, total and high density lipoprotein cholesterol and triglycerides. J. Lipid Res. 35, 1318-1328 (1994).

32. Simo, J. M. et al. Instability of lipoprotein(a) in plasma stored at -70 degrees C: effects of concentration, apolipoprotein(a) genotype, and donor cardiovascular disease. Clin. Chem. 47, 1673-1678 (2001).

33. Wieringa, G. et al. Changes in lipoprotein(a) levels measured by six kit methods during growth hormone treatment of growth hormone-deficient adults. Growth Horm. IGF Res. 10, 14-19 (2000).

34. Simonsson, M., Schmidt, C., Sigurdardottir, V., Helenius, M. \& Fagerberg, B. Life style habits such as alcohol consumption and physical activity in relation to serum apoB/apoA-I ratio amongst 64-year-old women with varying degrees of glucose tolerance. Jornal of Internal Medicine 262, 537-544 (2007).

35. Balogh, E. et al. Interaction between homocysteine and lipoprotein(a) increases the prevalence of coronary artery disease/myocardial infarction in women: a casecontrol study. Thromb. Res. 129, 133-138 (2012).

36. Undas, A. \& Ariens, R. A. Fibrin clot structure and function: a role in the pathophysiology of arterial and venous thromboembolic diseases. Arterioscler. Thromb. Vasc. Biol. 31, e88-99 (2011).

37. Dunn, E. J., Ariens, R. A. \& Grant, P. J. The influence of type 2 diabetes on fibrin structure and function. Diabetologia 48, 1198-1206 (2005).

38. Jorneskog, G., Hansson, L. O., Wallen, N. H., Yngen, M. \& Blomback, M. Increased plasma fibrin gel porosity in patients with Type I diabetes during continuous subcutaneous insulin infusion. J. Thromb. Haemost. 1, 1195-1201 (2003).

39. Alzahrani, S. H. et al. Gender-specific alterations in fibrin structure function in type 2 diabetes: associations with cardiometabolic and vascular markers. J. Clin. Endocrinol. Metab. 97, E2282-7 (2012).

40. van Giezen, J. J., Wahlund, G. Nerme \& Abrahamsson, T. The Fab-fragment of a PAI-1 inhibiting antibody reduces thrombus size and restores blood flow in a rat model of arterial thrombosis. Thromb. Haemost. 77, 964-969 (1997).

41. Undas, A., Stepien, E., Tracz, W. \& Szczeklik, A. Lipoprotein(a) as a modifier of fibrin clot permeability and susceptibility to lysis. J. Thromb. Haemost. 4, 973-975 (2006).

42. Undas, A., Kolarz, M., Kopec, G. \& Wieslawa, T. Altered fibrin clot properies in patients on long-term haemodialysis: relation to cardiovascular mortality. Nephrology, dialysis, transplantation: official publication of the European Dialysis and Transplant Association - European Renal Association 23, 2010 (2008).

43. Undas, A., Slowik, A., Wolkow, P., Szczudlik, A. \& Tracz, W. Fibrin clot properties in acute ischemic stroke: relation to neurological deficit. Thromb. Res. 125, 357-361 (2010)

44. Pera, J. et al. Fibrin clot properties in acute stroke: what differs cerebral hemorrhage from cerebral ischemia? Stroke 43, 1412-1414 (2012).

45. Alberti, K. G. \& Zimmet, P. Z. Definition, diagnosis and classification of diabetes mellitus and its complications. Part 1: diagnosis and classification of diabetes mellitus provisional report of a WHO consultation. Diabet. Med. 15, 539-553 (1998).

46. Prahl, U. et al. Slightly elevated high-sensitivity C-reactive protein (hsCRP) concentrations are associated with carotid atherosclerosis in women with varying degrees of glucose tolerance. Angiology 61, 793-801 (2010).

47. Ryndel, M. et al. The haptoglobin 2-2 genotype is associated with carotid atherosclerosis in 64-year old women with established diabetes. Clin. Chim. Acta 411, 500-504 (2010).

48. Fagerberg, B., Kellis, D., Bergstrom, G. \& Behre, C. J. Adiponectin in relation to insulin sensitivity and insulin secretion in the development of type 2 diabetes: a prospective study in 64-year-old women. J. Intern. Med. 269, 636-643 (2011).

49. Deming, W. E. in Statistical adjustment of data (Wiley, NY, 1943).

50. Pieters, M., Kotze, R. C., Jerling, J. C., Kruger, A. \& Ariens, R. A. Evidence that fibrinogen gamma' regulates plasma clot structure and lysis and relationship to cardiovascular risk factors in black Africans. Blood 121, 3254-3260 (2013). 
51. Leurs, J. et al. Different mechanisms contribute to the biphasic pattern of carboxypeptidase U (TAFIa) generation during in vitro clot lysis in human plasma. Thromb. Haemost. 89, 264-271 (2003).

52. Meltzer, M. E. et al. Venous thrombosis risk associated with plasma hypofibrinolysis is explained by elevated plasma levels of TAFI and PAI-1. Blood 116, 113-121 (2010).

53. Bjorquist, P., Ehnebom, J., Inghardt, T. \& Deinum, J. Epitopes on plasminogen activator inhibitor type-1 important for binding to tissue plasminogen activator. Biochim. Biophys. Acta 1341, 87-98 (1997)

54. Eriksson, H. et al. CPU inhibtion with AZD9684: profibrinolytic effects in acute patients. J Thromb Haemost 5, PS 367 (2007).

55. Undas, A. et al. Altered fibrin clot structure/function in patients with idiopathic venous thromboembolism and in their relatives. Blood 114, 4272-4278 (2009).

\section{Acknowledgments}

The authors thank the DIWA research team at Sahlgrenska University Hospital, Gothenburg, Sweden, special thanks to Brigitta Jannemark and Marie-Louise Ekholm for their highly professional work with the collection of data, blood sampling and biobanking. Thanks to Anders Thelin and Ann-Margret Lindquist, AstraZeneca for valuable discussions and advice, and to Peter Ceuppens, AstraZeneca, for proof-reading the manuscript.

\section{Author contributions}

M.M. did the statistical analysis and wrote the manuscript. I.K. designed research and wrote the manuscript. G.B. designed research and leads the DIWA study team. C.S. managed the database and edited the manuscript. A.L. designed and performed the CLA. L.M.H. and L.A.-F. designed and performed the Lp(a) analysis. D.G. contributed to the research and edited the manuscript. W.K. designed research and experiments and wrote the manuscript.

\section{Additional information}

Supplementary information accompanies this paper at http://www.nature.com/ scientificreports

Competing financial interests: M.M., I.K., A.L., L.A.-F., D.G. and W.K. were paid employees at AstraZeneca while working on this project/manuscript. G.B. is supported by grants from the Swedish Heart-Lung foundation. L.M.H. and C.S. had no competing interests.

How to cite this article: Månsson, M. et al. $\mathrm{Lp}(\mathrm{a})$ is not associated with diabetes but affects fibrinolysis and clot structure ex vivo. Sci. Rep. 4, 5318; DOI:10.1038/srep05318 (2014)

This work is licensed under a Creative Commons Attribution-NonCommercialNoDerivs 4.0 International License. The images or other third party material in this article are included in the article's Creative Commons license, unless indicated otherwise in the credit line; if the material is not included under the Creative Commons license, users will need to obtain permission from the license holder in order to reproduce the material. To view a copy of this license, visit http:// creativecommons.org/licenses/by-nc-nd/4.0/ 